\title{
Mapeamento de Indícios de Mediação em Ambiente Virtual de Ensino e Aprendizagem por Meio de Um Sistema de Mineração de Textos
}

\author{
Carlos Emilio Padilla Severo, Liliana Maria Passerino, José Valdeni de Lima \\ Programa de Pós-Graduação em Informática na Educação (PGIE) - Universidade \\ Federal do Rio Grande do Sul (UFRGS) - Porto Alegre - RS - Brasil \\ emilio.severo@gmail.com, liliana@cinted.ufrgs.br, valdeni@inf.ufrgs.br
}

\begin{abstract}
This paper presents a research about the mapping of mediation evidences to support the pedagogical intervention in AVEA. Text mining techniques were used and Bayesian inference mechanisms were employed to categorize the interactions. A case study was applied to validate the research. Data were collected from the textual interactions. The system generated graphs and reports about the levels of mediation. As a result, we highlight the production of qualitative information about the interactions, whose contribution was the mapping of control categories to aid in identifying the levels of student autonomy.
\end{abstract}

Resumo. Este artigo apresenta uma investigação sobre o mapeamento de indícios de mediação para apoio a mediação pedagógica em AVEA. Técnicas de mineração de textos foram utilizadas e mecanismos de inferência bayesiana foram empregados para categorização das interações. Um estudo de caso foi aplicado para validação da pesquisa. Os dados foram coletados a partir das interações textuais. O sistema gerou gráficos e relatórios acerca dos níveis das mediações. Como resultado, destaca-se a produção de informações qualitativas acerca das interações, cuja contribuição foi o mapeamento de categorias de controle para auxilio na identificação dos níveis de autonomia do estudante.

\section{Introdução}

A mediação pedagógica é um elemento importante no processo de construção do conhecimento. Nela, o professor se torna um agente essencial durante $o$ desenvolvimento da aprendizagem, visto que o mesmo deve intervir com o objetivo de promover aprendizagem e reflexão dos alunos. Nesse sentido, o professor como mediador da aprendizagem não exerce somente a função de facilitador no processo de construção do conhecimento, através da execução de estratégias que visem contornar problemas na aquisição de novos conceitos, mas também deve agir para auxiliar na aquisição de autonomia por parte do aluno [Wertsch 1999]. Durante a mediação o professor desenvolverá estratégias para contornar possíveis problemas e adaptar-se às distintas necessidades e particularidades de cada aluno, em busca das suas intenções pedagógicas. Esta premissa é válida tanto na modalidade de ensino presencial como a distância. Entretanto, as particularidades e diferenças inerentes aos espaços de ensino presencial e virtual fazem com que a mediação não seja uma mera transposição. Sendo assim, esta pesquisa visou abordar questões relacionadas ao apoio da mediação em ambientes de educação online com uso de tecnologias computacionais. 
Dessa forma, o foco da pesquisa foi o levantamento de indícios sobre mediação pedagógica em Ambiente Virtual de Ensino e Aprendizagem (AVEA). Sendo assim, realizou-se um acompanhamento das mediações ocorridas em um ambiente educacional online, a partir das interações textuais realizadas pelos participantes. Com este acompanhamento visou-se identificar os níveis das mediações realizadas. Para isso, recorreu-se à tecnologia computacional de mineração de textos para auxiliar no mapeamento das categorias de interações entre os participantes através de uma extensão ao ambiente Moodle. A extensão visou à verificação de possibilidades de acompanhamento do desenvolvimento das mediações das aprendizagens dos estudantes, a partir de interações realizadas no ambiente. Com isso, auxiliando-se professores e tutores com elementos para suas estratégias de intervenções pedagógicas no AVEA.

De forma geral, o acompanhamento da participação dos alunos em atividades nos AVEA fornece subsídios ao professor para a intervenção pedagógica durante o desenvolvimento da aprendizagem dos estudantes. Porém, na forma como um AVEA é estruturado na atualidade, a coleta de tais indícios constitui um grande desafio devido ao volume de informações geradas a partir das interações dos usuários.

Em geral, percebeu-se que esse acompanhamento automatizado dos estudantes em AVEA é realizado levando-se em conta aspectos quantitativos, tais como: número de acessos, número de interações em fóruns ou tarefas concluídas sem levar em conta aspectos qualitativos, sobre o que os estudantes estão efetivamente trocando (conteúdo das interações). Tal fato, pode-se observar a partir de trabalhos realizados sobre o tema. Como no trabalho de [Ferreira et. al. 2003], onde foi apresentada uma interface para registro e análise de avaliações realizadas pelo professor durante o desenvolvimento de um curso. Tal investigação possibilitou o registro das observações do professor acerca da avaliação formativa dos alunos, entretanto de forma manual. Já em [Romani 2000] foi apresentada uma ferramenta para auxílio no acompanhamento das interações em AVEA, através do mapeamento entre alunos; e, alunos com professores, permitindo a quantificação e visualização gráfica das relações entre os indivíduos a partir de suas interações. Em [Bassani e Behar 2006] o foco foi quantificar acessos e número de contribuições postadas em um AVEA, apresentando relatórios relativos às interações de cada aluno.

$\mathrm{Na}$ presente pesquisa, como diferencial em relação aos trabalhos citados, investigou-se como construir e aplicar um sistema de mineração automática de dados o qual visa auxiliar na redução da carga de trabalho do professor durante suas atividades de mediação pedagógica em AVEA. Neste sentido, para compreensão da mediação como processo pedagógico, utilizou-se a teoria sócio-histórica como base epistêmica, através das obras de [Vygotsky 2007], bem como, autores contemporâneos da mesma linha como [Werstch 1999], [Gallimore e Tharp 1996] e [Diaz et. al. 1993]. Os quais representaram o alicerce para os estudos conduzidos nesta investigação sobre atividades de mediação em AVEA.

Um dos problemas atuais dos pesquisadores sócio-históricos é acompanhar e compreender o processo de mediação que culmina na internalização dos instrumentos e signos de mediação pelo sujeito menos experiente, e que é o principal mecanismo que explica a aprendizagem na teoria sócio-histórica. Pesquisadores ao longo de décadas têm procurado identificar formas de acompanhamento do processo de mediação a partir de diferentes indícios cognitivos e semióticos [Diaz et. al. 1993], [Gallimore e Tharp 
1996] e [Wertsch 1999]. Nesta pesquisa, utilizaram-se inicialmente as categorias de mediação apontadas nos trabalhos de [Dias et. al. 1993] e, posteriormente ampliadas por [Passerino e Santarosa 2008], com validação na área de AVEA no trabalho de [Koch et. al. 2009], o qual mapeou indícios de mediação nos registros produzidos por cursos na modalidade EAD. Resumidamente, as categorias de mediação são:

- Controle: externo ao sujeito, realizado pelo sujeito mais experiente e pode assumir duas dimensões: direta ou indireta. $\mathrm{O}$ controle direto verifica-se através de ordens, diretivas e perguntas diretivas. Já o controle indireto constata-se através de perguntas perceptivas, conceituais e procedimentais, culminando no afastamento físico (o sujeito mais experiente somente observa o menos experiente). $\mathrm{O}$ gradual afastamento físico do sujeito mais experiente no processo de mediação da aprendizagem visa levar o estudante a entrar no nível de autocontrole.

- Autocontrole: é a realização, por parte do estudante, de uma ação esperada obedecendo a um tutor internalizado. Ou seja, a figura do sujeito mais experiente que era real e externo no processo de controle, agora é interna, mas ainda existe como outro sujeito no aparato cognitivo do estudante.

- Auto-regulação: esta categoria não pode ser observada de forma direta, pois a mesma acontece internamente ao sujeito, mas considera-se que o sujeito está na categoria de auto-regulação quando organiza, planeja e executa a ação sem intervenção de nenhum mediador externo. Assim, a auto-regulação é o plano de ação concebido pelo sujeito que se converte no seu próprio tutor.

A investigação descrita neste artigo está inserida no projeto MEDIATEC ${ }^{1}$. Sendo assim, buscou-se investigar como a mediação do professor em AVEA pode ser compartilhada por uma tecnologia de apoio às atividades de intervenção pedagógica, visando aliviar o trabalho do professor. Para isso, o projeto MEDIATEC envolve uma pesquisa interdisciplinar que alia elementos da área de interação social, educação a distância, sistemas inteligentes, aprendizagem de máquina e modelos cognitivos. $\mathrm{O}$ projeto MEDIATEC já produziu alguns resultados expressivos os quais são descritos nos trabalhos de [Gluz et. al. 2008] [Passerino e Santarosa 2008] [Koch at. al. 2009] [Raminelli et. al. 2009] [Severo et. al. 2011a] [Severo et. al. 2011b [Severo et. al. 2009].

\subsection{O problema de pesquisa e objeto de estudo}

A investigação abordou possibilidades que as tecnologias de informação e comunicação (TIC) podem acrescentar a um AVEA, visando melhores condições ao processo de mediação pedagógica. Para tanto, levantaram-se questões que serviram de bases condutoras para identificação e delimitação do problema de pesquisa: a) como o processo de mediação pode ser percebido e gerenciado nos diferentes espaços de interação do AVEA? B) De que forma a mineração de textos pode ser utilizada para mapeamento de interações e levantamento de indícios de mediação em AVEA? C) De que forma podem ser disponibilizadas informações decorrentes do mapeamento para auxilio ao professor na tarefa de mediação pedagógica em um AVEA?

\footnotetext{
${ }^{1}$ O MEDIATEC é um projeto apoiado financeiramente pelo CNPQ que visa verificar como os ajustes de mediação pedagógica podem ser realizados no contexto educativo na modalidade de ensino a distância.
} 
Sendo assim, chegou-se à identificação do problema de pesquisa: de que forma a tecnologia de mineração de textos e a epistemologia sócio-histórica podem fornecer elementos que apoiem a mediação pedagógica em ambientes virtuais de ensino e aprendizagem?

Levando-se em consideração o objeto de estudo apresentado, esta pesquisa foi uma abordagem de ciência aplicada, pois visou à exploração e experimentação de conhecimentos sobre fenômenos de natureza tecnológica e sócio-cultural. Dessa maneira, pode-se identificar a interdisciplinaridade do trabalho, através de sua relação com duas grandes áreas do conhecimento: ciências exatas e ciências sociais. No ramo das ciências exatas está a computação, que apoiou o trabalho através de suas tecnologias. Já no ramo das ciências sociais está a educação e psicologia cuja influência no trabalho está na base teórica sobre mediação pedagógica e aprendizagem.

No âmbito da relevância, acredita-se que a pesquisa apresentou um diferencial quanto à aplicação de aspectos teóricos envolvidos na mediação da aprendizagem em um ambiente distinto do contexto convencional que é a sala de aula presencial. Trata-se de um trabalho que buscou a identificação e gerenciamento do processo de mediação pedagógica na modalidade de EAD, apoiada em AVEA. Originalidade diz respeito à busca por novos resultados ainda não disseminados no meio científico ou profissional. Em pesquisas preliminares, não foi identificado algum mecanismo de apoio a mediação pedagógica nos AVEA atuais, que apresentem elementos indicativos sobre $o$ desenvolvimento da aprendizagem dos estudantes ao mediador, a partir do mapeamento das dinâmicas de mediação decorrentes de interações textuais dos participantes do curso no ambiente online.

\subsection{Os objetivos da pesquisa}

O objetivo geral foi investigar como um sistema de mineração permite mapear o conteúdo de interações em AVEA, identificando níveis de mediação e visando o levantamento de elementos de apoio à mediação pedagógica ao professor e tutor de um curso online. Com base no objetivo geral do trabalho, os seguintes objetivos específicos serviram como linhas condutoras da investigação:

a) Identificar e estudar técnicas de mineração de texto para mapeamento da mediação dos alunos a partir de sessões de interação em AVEA.

b) Propor um sistema para apoio à mediação pedagógica do professor em ambientes virtuais de ensino e aprendizagem.

c) Representar graficamente as ações de mediação pedagógica extraídas de sessões de interação entre o professor mediador e os alunos.

\section{A investigação}

Quanto aos procedimentos metodológicos, a pesquisa foi de desenvolvimento tecnológico para exame do ambiente de aprendizagem, visando à compreensão da mediação pedagógica em cunho qualitativo para validação da proposta tecnológica. A utilização de um estudo de caso real para aplicação de uma pesquisa empírica de natureza qualitativa e exploratória foi justificável nesta pesquisa, tendo em vista a análise dos impactos pedagógicos da implantação de inovações tecnológicas em ambientes virtuais de ensino e aprendizagem. 
O estudo de caso foi desenvolvido com participantes de uma disciplina do curso de mídias na educação do Centro Interdisciplinar de Novas Tecnologias na Educação CINTED, da Universidade Federal do Rio Grande do Sul. No período de primeiro de agosto de dois mil e onze até trinta e um de outubro de dois mil e onze. Durante o desenvolvimento da disciplina de Prática pedagógica em ambiente apoiado pela TIC. A investigação foi desenvolvida em um AVEA contendo dados provenientes do curso online. Como técnicas de coleta de dados utilizaram-se:

- Observações diretas de processos envolvidos na implantação do sistema emediation no AVEA Moodle. Assim como, o comportamento do mesmo durante a captura dos indícios de mediação. Com a utilização da observação buscamos a identificação dos indícios que comprovem a ocorrência de mediação no ambiente através da identificação do nível de intervenção pedagógica necessária aos alunos com base nas categorias de mediação levantadas nos trabalhos de [Passerino e Santarosa 2008], [Koch et. al. 2009] e [Diaz 1993], com a utilização de algoritmos de mineração de textos.

- Entrevista semi-estruturada com a tutora da disciplina. Adotou-se a técnica de entrevista semi-estruturada, visando-se respostas mais abrangentes aos questionamentos. Com a utilização da entrevista, buscaram-se elementos que pudessem ser úteis para análise do impacto pedagógico da aplicação do sistema de mineração de indícios de mediação no ambiente online.

Para validação da pesquisa, realizou-se uma triangulação entre a entrevista, as observações diretas do pesquisador e as informações geradas pelo sistema sobre as mediações realizadas no ambiente. A seguir, discorre-se sobre o processo investigativo.

A investigação envolveu as etapas de treinamento e teste dos modelos de inferência aplicados na pesquisa; especificação conceitual, desenvolvimento e teste do sistema e-mediation; e a aplicação do sistema em um estudo de caso, bem como, a análise dos resultados.

O treinamento e teste do modelo de mineração de textos utilizado pelo sistema e-mediation formam a primeira etapa na condução da investigação, pois visou à aplicação de um processo de mineração de textos a fim de se obter um modelo a ser aplicado em dados provenientes das interações dos sujeitos. Dois modelos foram treinados e testados. O modelo do aluno e modelo do mediador. Com o modelo do aluno, visou-se a indução do nível de mediação da aprendizagem do mesmo em um determinado período do curso. Já o modelo do mediador visou à indução do nível de controle exercido pelo mesmo durante suas mediações no decorrer do curso. Sendo assim, utilizaram-se dados previamente classificados por especialistas de forma manual. Tal tarefa inicial foi primordial para o treinamento dos modelos de aprendizagem supervisionada utilizados nesta pesquisa. $O$ processo de identificação manual das categorias de mediação é descrito no trabalho de [Koch et. al. 2009]. Também foi utilizado o processo de mineração de textos descrito no trabalho de [Raminelli et. al. 2009], no qual foram avaliados previamente os desempenhos de algoritmos de classificação bayesiana para levantamento das categorias de mediação, viabilizando o desenvolvimento do sistema e-mediation. Ambos os trabalhos citados constituem o escopo do projeto MEDIATEC, portanto, parte constituinte da investigação abordada nesta pesquisa. 
A próxima etapa foi a especificação conceitual, desenvolvimento e testes do sistema e-mediation. Nesta segunda etapa da investigação foi realizada a modelagem conceitual e de domínio do sistema de mediação, destacando-se seus requisitos e funções desejadas. Bem como, aspectos tecnológicos envolvidos na construção do sistema e sua integração com o ambiente Moodle, através de um modelo de componentes. A Figura 1 ilustra as principais funções do sistema e-mediation, através de um diagrama de casos de uso (a) e um diagrama de componentes que apresenta as tecnologias utilizadas no desenvolvimento do sistema.

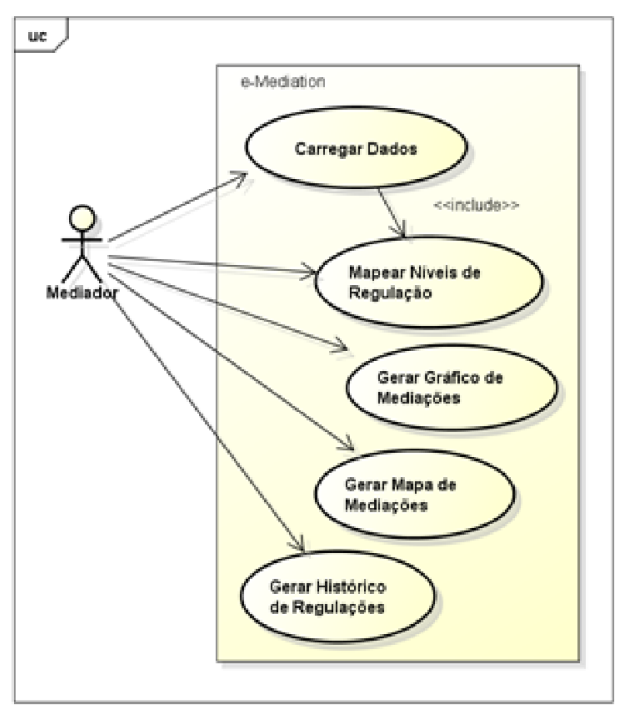

(a)

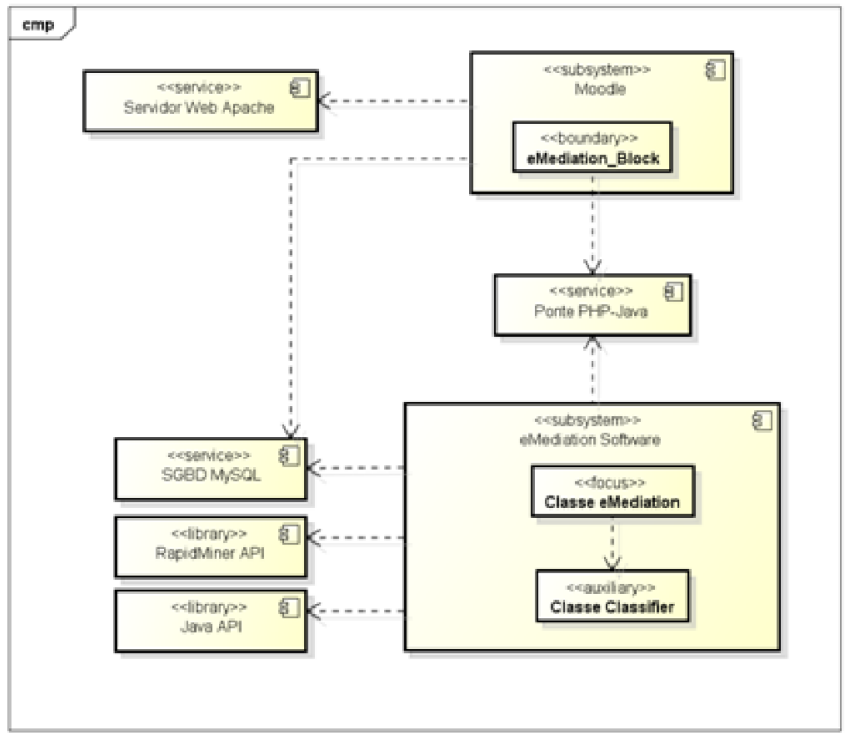

(b)

Figura 1. Casos de uso (a) e modelo de componentes (b) do sistema emediation. Fonte: própria autoria.

Por fim, procedeu-se a etapa de aplicação do sistema no estudo de caso e análise dos resultados. Esta fase foi o foco do trabalho de investigação, cuja aplicação das funcionalidades do e-mediation visou o mapeamento de interações e identificação dos níveis de mediação da aprendizagem ao longo do desenvolvimento da disciplina. Tal etapa culminou na análise dos resultados e validação do trabalho de investigação.

\section{Análise e discussão dos resultados}

Para que as questões norteadoras da pesquisa possam ser respondidas, bem como, os objetivos serem alcançados, descrevem-se os resultados sobre os seguintes pontos de vista:

- Uma análise da aplicação do sistema e-mediation.

- Uma análise do próprio pesquisador sobre as interações realizadas no ambiente.

- Uma análise da tutora a partir da entrevista.

Neste estudo foram realizados mapeamentos de dados em três períodos no decorrer da disciplina. Com isso, pretendeu-se realizar um acompanhamento da evolução das interações dos estudantes, buscando-se analisar os níveis de mediação ao longo da disciplina. As análises foram realizadas sobre as interações de quatro sujeitos e da tutora da disciplina. 
A análise dos resultados da aplicação do e-mediation foi realizada pela interpretação das informações geradas pelos gráficos e relatórios do sistema, ilustrados através da Figura 2. A ilustração apresenta a interface do (a) gráfico de nível das mediações dos participantes (visão geral das categorias de mediação mapeadas a partir de todas as interações dos participantes); uma prévia de um (b) mapa de mediações do estudante (representando as mediações entre estudante-tutor e estudante-estudante durante o período de mapeamento); e, um (c) histórico de regulação da aprendizagem do estudante (refletindo a evolução da aprendizagem do estudante durante os três períodos de mapeamento, a partir das categorias de mediação mapeadas pelo sistema).

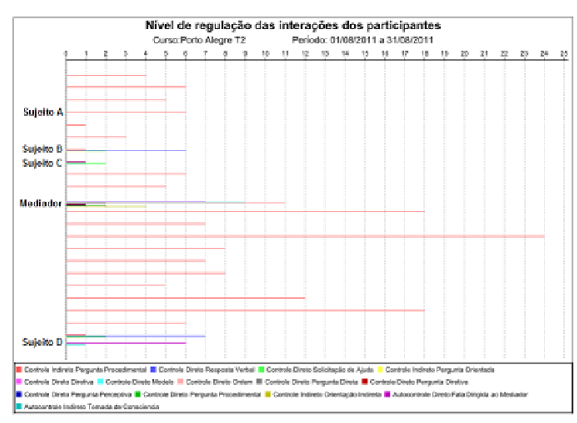

(a)

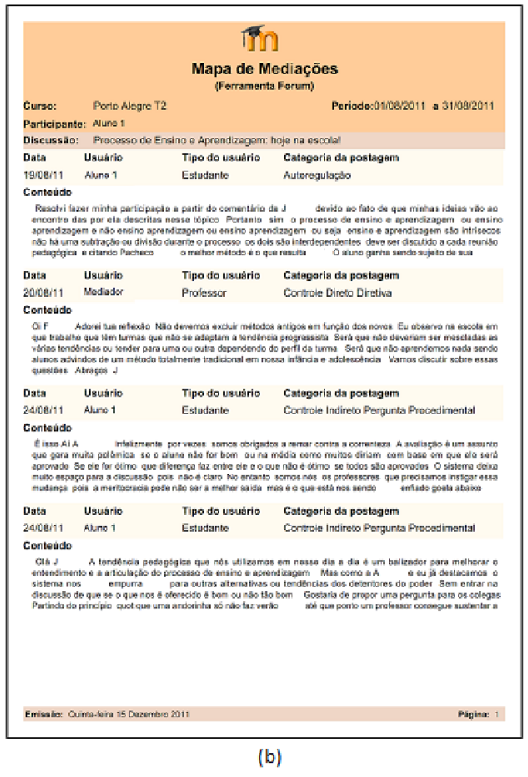

(b)

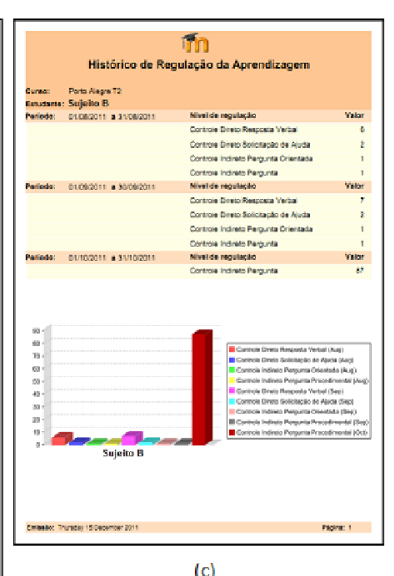

(c)

Figura 2. Gráfico de Nível de Mediação (a), Mapa de mediações (b) e histórico de regulação da aprendizagem (c). Fonte: própria autoria.

A Tabela 1 apresenta os resultados apontados pelo sistema e-mediation. Neste quadro, pode-se observar um resumo quantitativo das interações dos participantes no decorrer dos períodos de mapeamento de dados. Bem como, um panorama qualitativo acerca do nível de regulação das interações. A seguir, apresentam-se a análise dos resultados apresentados pelo sistema e-mediation. Será apresentada a análise de somente dois sujeitos da pesquisa. Embora no estudo de caso tenham participado quatro sujeitos. Como critério de escolha do sujeitos, optou-se pelo sujeito de menor grau de autonomia levantado pelo sistema e o sujeito de maior grau de autonomia. A análise detalhada dos quatro sujeitos participantes pode ser encontrada em [Severo 2012].

O sistema de mapeamento identificou que o sujeito A recorreu muito a interações de controle indireto na forma de perguntas orientadas a tutora da disciplina. Também ocorreram interações de controle direto do tipo resposta verbal. Nos gráficos e relatórios gerados pelo sistema, pode-se observar um grau de evolução nas interações da estudante. $\mathrm{O}$ que identifica que a estudante necessitou de ações de intervenção de controle por parte da mediadora da disciplina. 
Tabela 1. Resultado Quantitativo e qualitativo do mapeamento das interações. Fonte: própria autoria.

\begin{tabular}{lrrrl}
\hline & \multicolumn{3}{c}{$\begin{array}{c}\text { Quantidade de interações } \\
\text { (2011) }\end{array}$} & Categoria de Mediação \\
SAgnificativa
\end{tabular}

A análise do pesquisador constatou os resultados apontados pelo sistema de mapeamento. A estudante apresentou um bom desempenho em suas atividades, com algumas dúvidas decorrentes da utilização da ferramenta de escrita colaborativa, recorrendo para isso a interações na forma de solicitação de ajuda. O que pode ser observado nas sessões síncronas na ferramenta chat e na leitura do diário da estudante. Em alguns momentos foram constatadas interações de auto-regulação, confirmando o ótimo desempenho da estudante. Entretanto, encontrando algumas dificuldades no uso de recursos do ambiente no decorrer de suas tarefas. O que levou ao mapeamento de uma maior incidência de controle indireto. A entrevista com a tutora da disciplina confirmou o que foi constatado pelo sistema e pela análise do pesquisador. Durante o relato sobre o desempenho a tutora informou que estudante teve um início de dificuldades inerentes de sua inexperiência com a utilização do AVEA. A conclusão da análise do sujeito A é que a estudante se encontra em um nível de controle indireto, com certa autonomia no desenvolvimento de suas atividades. Porém, a estudante necessitou de auxílio da tutora devido a alguma insegurança com a manipulação dos recursos do ambiente virtual.

Já o sujeito D foi o mais interativo dos quatro sujeitos analisados nesta investigação. O sistema apontou que o estudante foi muito participante desde o início da disciplina. As interações mapeadas pelo sistema identificaram um alto nível de autonomia do estudante. Notou-se a ocorrência de muitas contribuições na categoria de controle indireto e autocontrole. A análise do pesquisador constatou as informações apontadas pelo sistema. O aluno foi muito participativo e crítico, buscou sempre contribuir e enriquecer as discussões para questões levantadas no fórum. A análise dos registros no diário confirma a autonomia do estudante no desenvolvimento de sua aprendizagem. Notaram-se poucas interações de solicitação de ajuda. A grande maioria foram interações de respostas verbais e fala dirigida ao mediador. A tutora confirmou os resultados do sistema. De acordo com a tutora o sujeito D foi o estudante de melhor desempenho da turma. A tutora citou que o estudante apresentou um elevado grau de autonomia e conhecimentos prévios sobre ambiente e muita experiência profissional.

Dessa forma, a partir dos resultados do sistema e análises realizadas, conclui-se que o sujeito D se encontra em um nível de autocontrole. Visto que a própria tutora informou que poucas ações de intervenção pedagógica foram realizadas no decorrer da disciplina, pois o estudante apresentou um excelente desempenho. 


\section{Considerações finais}

A título de conclusão acerca do trabalho desenvolvido nesta pesquisa, cumpre destacar que o mesmo envolveu dois eixos investigativos. Em um primeiro momento abordou-se questões sobre a utilização e combinação de tecnologias computacionais para concepção de um sistema para o mapeamento de indícios de mediação da aprendizagem de estudantes em AVEA. Em um segundo momento a aplicação do sistema na detecção de indícios de mediação da aprendizagem a partir de um estudo de caso.

Ambos os eixos investigativos convergiram para a abordagem metodológica aplicada nesta pesquisa, onde os subsídios teóricos foram combinados com tecnologias computacionais, visando às respostas para as questões norteadoras da investigação.

A resposta a primeira questão é a compreensão do processo de mediação com base na teoria sócio-histórica, a partir de uma abordagem que permita o mapeamento do processo de mediação em AVEA. Assim, pode-se perceber como o processo de mediação ocorre nos espaços de interação. O processo de mediação foi gerenciado nos diferentes espaços de interação do ambiente com o emprego do sistema desenvolvido nesta pesquisa. $\mathrm{O}$ e-mediation provê um conjunto de mecanismos e uma estrutura lógica que permite o mapeamento das ações de mediação e níveis de mediação da aprendizagem dos estudantes no ambiente. A resposta a segunda questão norteadora é a utilização da tecnologia de mineração de textos, mais precisamente a combinação do algoritmo de inferência bayesiana em um processo de classificação das interações em categorias de mediação. Já a terceira questão é respondida a partir dos relatórios e gráficos gerados pelo sistema.

A partir das respostas às questões norteadoras chegou-se a uma resposta ao problema de pesquisa: de que forma a tecnologia de mineração de textos e a epistemologia sócio-histórica podem fornecer elementos que apoiem a mediação pedagógica em ambientes virtuais de ensino e aprendizagem?

As informações geradas pelo sistema e-mediation mostraram-se eficientes para o professor e tutor obterem um panorama qualitativo sobre como ocorre o processo de mediação e desenvolvimento da aprendizagem do estudante ao longo de um período. Tais constatações foram comprovadas pela triangulação das análises realizadas nesta pesquisa, quando foram confrontadas as diferentes fontes de coleta.

Portanto, com o exposto nestas considerações finais, acredita-se que os objetivos foram alcançados com a conclusão deste trabalho investigativo. Através da utilização de um sistema de mapeamento de indícios de mediação com adoção da tecnologia de mineração de textos, torna-se possível o levantamento de elementos para apoio ao processo de mediação pedagógica em AVEA.

\section{Referências}

Bassani, P. S., Behar, P. A (2006). Análise das interações em ambientes virtuais de aprendizagem: uma possibilidade para avaliação da aprendizagem em EAD. Revista Novas Tecnologias na Educação, v. 4, nº 1.

Diaz, R., Neal, C., Amaya-Williams, M (1993). Orígenes sociales de la autorregulación. In Moll, L.C (comp). Vygotksy y la educación: connotaciones y aplicaciones de la psicologia sociohistórica en la educación. Buenos Aires: Aique. 
Ferreira, T. B., Otsuka, J. L., Rocha, H. V (2003). Interface para Auxílio à Avaliação Formativa no Ambiente TelEduc, XIV Simpósio Brasileiro de Informática na Educação - SBIE 2003, Rio de Janeiro, UFRJ, p. 160-169.

Gallimore, R., Tharp, R. (1996). O pensamento educativo na sociedade: ensino, escolarização e discurso escrito. In: MOLL, Luis C. Vygotsky e a educação: implicações pedagógicas da psicologia sócio-histórica. Porto Alegre: Artes Médicas.

Gluz, J. C., Passerino, L. M., Vicari, R. M. (2008). Um modelo formal para processos de mediação em AVAs. In: XIX SBIE - simpósio brasileiro de informática na educação, Fortaleza, Ceará.

Koch, S. H. S., Maciel, M. C. P., Passerino, L. M. (2009). The mediation in distance learning: possibilities of mapping the signs. Proceedings of $9^{\text {th }}$ WCCE 2009, Bento Gonçalves, Brasil, pp. 94.

Passerino, L. M., Santarosa, L. M. C. (2008). Learning Environments: processes of interaction and mediation. Computers and Education, v. 51, p. 385-402.

Raminelli, A., Gluz, J. C., Passerino, L. M. (2009). Modelo Bayesiano de Classificação de Categorias de Mediação em Ferramentas de EAD. São Leopoldo: UNISINOS, 2009. Relatório técnico do projeto MEDIATEC, Universidade Federal do Rio Grande do Sul e Universidade do Vale do Rio dos Sinos.

Romani, L. A. (2000). Intermap: ferramenta para visualização da interação em ambientes de educação a distância na web. Dissertação (Mestrado em Computação), IC/Unicamp, Campinas.

Severo, C. E. P. (2012). E-mediation: mapeamento de indícios de mediação por meio de um sistema de mineração de textos. Tese (Doutorado em Informática na Educação), PGIE/UFRGS, Porto Alegre.

Severo, C. E. P., Passerino, L. M., Gluz, J. C., Raminelli, A. (2011a) Mediação pedagógica em ambientes virtuais de ensino e aprendizagem através de agentes de mineração de dados educacionais. Informática na Educação: Teoria e Prática, v. 14, n. 2.

Severo, C. E. P., Passerino, L. M., Lima, J. V. (2011). Uma ferramenta de apoio à mediação pedagógica para o ambiente Moodle. In: Primer MoodleMoot Uruguay, 2011, Montevideo. El primer MoodleMoot Uruguay, 2011b. v. 1. p. 1-5.

Severo, C. E. P., Passerino, L. M., LIMA, J. V. (2009). E-mediation: a proposal of a miner tool for virtual learning environment. In: IFIP World Conference on Computers in Education, 2009, Bento Gonçalves. Technology and Education for a Better World. Porto Alegre : WCCE, 2009. p. 233-236.

Vygotsky, L. S. (2007). A Formação Social da Mente. São Paulo: Martins Fontes.

Wertsch, J. (1999). La mente em acción. Buenos Aires: Aique. 